\title{
Average plasma sheet polytropic index as observed by THEMIS
}

\author{
Dennis Frühauff, Johannes Z. D. Mieth, and Karl-Heinz Glassmeier \\ Institut für Geophysik und extraterrestrische Physik, Braunschweig, Germany \\ Correspondence to: Dennis Frühauff (d.fruehauff@tu-bs.de)
}

Received: 24 October 2016 - Revised: 23 December 2016 - Accepted: 10 February 2017 - Published: 24 February 2017

\begin{abstract}
Multi-spacecraft data from the years 2008 to 2015 of the THEMIS mission particularly in the near-Earth plasma sheet are used in order to empirically determine the polytropic index in the quiet and active time magnetotail. The results of a number of previous studies in the 1990s can be confirmed. An analysis of the total database, although showing poor correlation, results in an average polytropic index of $\gamma=1.72$. The active time plasma sheet is well correlated with an average $\gamma=1.49$. However, the data scattering suggests that the analysis of the data in total is not adequate. In order to reduce the timescales, individual spacecraft orbits are analyzed, giving a broad distribution of polytropic indices throughout the plasma sheet. The major part of the distribution falls in a range between $\gamma=0.67$ and $\gamma=2$. Our results indicate a variety of thermodynamic processes in the magnetotail and an all-time presence of heat exchange of the plasma. A description of the plasma sheet using an equation of state with a single $\gamma$ is probably inadequate. This necessitates the application of more sophisticated approaches, such as a parametrization of the heat flux vector in magnetohydrodynamic equations or a superposition of polytropic indices.
\end{abstract}

Keywords. Magnetospheric physics (magnetotail; plasma sheet)

\section{Introduction}

The plasma sheet is one of many important regions in the Earth's magnetotail. A large variety of dynamic processes are located in or connected to this region, such as magnetohydrodynamic waves, reconnection, fast plasma flows and convection, (e.g., Nishida, 2000). Theoretical models and numerical simulations, especially in terms of magnetohydrodynamic theory, require the closure of the system of moment equations. Usually, this is done by assuming a relationship between plasma pressure, $P$, and number density, $n$, the equation of state. Following Siscoe (1983) the general equation for the scalar pressure is

$P=\alpha n^{\gamma}$,

with $\alpha$ being a constant related to the specific entropy of a plasma sheet flux tube and $\gamma$ being the polytropic index. Further assuming a relationship between pressure, density, and temperature, $T$, similar to an ideal gas, $P=n k_{\mathrm{B}} T$, the second polytropic equation gives

$T=\alpha^{\prime} n^{\gamma-1}$.

In classical thermodynamics, the polytropic index is related to the degrees of freedom, $f$, of the magnetospheric plasma:

$\gamma=(f+2) / f$.

Since the plasma can have at most $f=3$ degrees of freedom, it follows that $\gamma \leq 5 / 3$ (Baumjohann and Paschmann, 1989; Zhu, 1990; Borovsky et al., 1998). Different thermodynamic scenarios arise from different values of $\gamma$ :

$$
\begin{aligned}
\gamma & =0, \text { isobaric, } \\
\gamma & =1, \text { isothermal, } \\
\gamma & =5 / 3, \text { adiabatic, } \\
(\gamma & =\infty, \text { isochoric), } \\
\text { and } & \\
\gamma & <5 / 3, \text { energy loss (cooling), } \\
\gamma & >5 / 3, \text { heating. }
\end{aligned}
$$

In an ideal system if (1) convection is reversible (i.e., particles are not lost from a flux tube); (2) apart from compression, no energy is transferred; and, (3) viscous effects are negligible, the system will behave adiabatically (Borovsky et al., 1998), which is the usual idealization in magnetohydrodynamic models and simulations. 
The availability of in situ satellite measurements has initiated several studies aiming the determination of the polytropic index of the Earth's plasma sheet over the past 30 years. Both modeling approaches but especially observational analyses have been performed.

Kivelson and Spence (1988) require a polytropic index of $\gamma=2 / 3$ to ensure the stability of their magnetotail model. Baumjohann and Paschmann (1989) have used AMPTE/IRM data of density and pressure in the plasma sheet and found that the tail behaves adiabatic in general and especially during magnetospheric active times. During quiet times they inferred $\gamma<5 / 3$, indicating cooling mechanisms and the absence of a magnetotail ground state. Huang et al. (1989) performed analyses on ISEE1/LEPEDEA data for the quiettime plasma sheet. They found density and temperature to be anti-correlated, i.e., $\gamma<1$, therefore being in contrast to the results of Baumjohann and Paschmann (1989). The total dataset gives $\gamma=0.6$. Spence and Kivelson (1990) provide a simple model accounting in parts for the differences between the two abovementioned studies by making the polytropic index a function of the midnight meridian in a magnetotail of finite width. Liu and Rostoker (1990) attempt to explain the differences between Baumjohann and Paschmann (1989) and Huang et al. (1989) with the measurement positions and selection criteria. They relate the Huang et al. (1989) results to initially hot flux tubes being loaded with cool ionospheric plasma that increases the plasma density while decreasing its temperature. Zhu (1990) further develops this explanation by outlining that the assumption of constant specific entropy of flux tubes for the complete dataset is not valid, since it crucially depends on the flux tube's past. Therefore, scattering is introduced in the variables. Zhu (1990) further points out that the usage of $n-P$ or $n-T$ analysis will influence the results as (1) the term temperature is not well defined for non-Maxwellian distributions and (2) the scattering is enhanced in the $n-T$ diagram through the computation of $T \propto P / n$. A reanalysis of ISEE1/FPE data in the $n-P$ domain for individual spacecraft orbits gives $1<\gamma<5 / 3$ using a plasma sheet criterion of $\beta=P /\left(B^{2} /\left(2 \mu_{0}\right)\right)>0.2$, first introduced by Baumjohann et al. (1988). Goertz and Baumjohann (1991), using AMPTE/IRM data as well but including the plasma sheet boundary layer, find a quiet time $\gamma=4 / 3$ and active time $\gamma=5 / 3$. They interpret their results as the presence of a mixture of bubbles and blobs and lead the partial contradiction of previous studies back to the choice of data selection criteria. Borovsky et al. (1998) compare plasma sheet data of ISEE2 in near-Earth, midtail, and downtail regions and emphasize the necessity to analyze the radial dependence of datasets. Following suggestions of Goertz and Baumjohann (1991) to use only neutral sheet/midnight data they find an average $\gamma=1.52$. Pang et al. (2015) use CLUSTER data of the central plasma and find a wide variety of polytropic indices ranging from 0.1 to 1.8 . Active- and quiettime results are very similar.
Table 1. THEMIS tail science phases used in this work.

\begin{tabular}{lrr}
\hline Stage & Start & End \\
\hline 3 & $2007 / 15 / 12$ & $2007 / 15 / 12$ \\
7 & $2008 / 15 / 12$ & $2008 / 15 / 12$ \\
11 & $2010 / 02 / 03$ & $2010 / 02 / 03$ \\
15 & $2011 / 16 / 03$ & $2011 / 16 / 03$ \\
19 & $2012 / 13 / 04$ & $2012 / 13 / 04$ \\
23 & $2013 / 11 / 06$ & $2013 / 11 / 06$ \\
27 & $2014 / 29 / 06$ & $2014 / 29 / 06$ \\
31 & $2015 / 15 / 08$ & $2015 / 15 / 08$ \\
\hline
\end{tabular}

In this work THEMIS and ARTEMIS data from eight intervals when THEMIS/ARTEMIS were located in their predefined tail science phases between 2008 and 2015 are used to revisit the in situ determination of the polytropic index in the central plasma sheet. Please refer to Table 1 for the list of intervals. The paper is organized as follows: Sect. 2 will introduce the data selection and orbital coverage, Sect. 3 presents key elements of the resulting database, and Sect. 4 will summarize the results.

\section{Data selection and analysis procedure}

The Time History of Events and Macroscale Interactions During Substorms (THEMIS) mission launched five identical spacecraft in 2007 into near-equatorial orbits around Earth (Angelopoulos, 2008). At present almost 9 years of data are available to the scientific community. The precessing perigee of the spacecraft constellation (in geocentric coordinate systems) accounts for seasonal science phases located in the dayside, duskside, dawnside, and tail regions of the magnetosphere. Here, the focus is on the tail science stages as shown in Table 1. Of these stages, 03, 07, and 11 provide near-Earth and midtail measurements between $-9 R_{\mathrm{E}}$ and $-30 R_{\mathrm{E}}$ downtail distance of five spacecraft. Later, two of the probes were lifted into a lunar orbit as the ARTEMIS mission. Consequently, these two spacecraft were then located around geocentric distances of about $60 R_{\mathrm{E}}$ (Angelopoulos, 2011).

Data from three instruments on board the spacecraft are used: the fluxgate magnetometer (FGM) provides magnetic field data at $\approx 4 \mathrm{~s}$ resolution (Auster et al., 2008). Particle data are provided by combined measurements of the electrostatic analyzer (ESA) in the $\leq 30 \mathrm{keV}$ range (McFadden et al., 2008a, b) and the solid-state telescope (SST) in the $25 \mathrm{keV}$ to $6 \mathrm{MeV}$ range (Angelopoulos, 2008), providing particle moments at spin $(\approx 3 \mathrm{~s})$ resolution. Position data are given in geocentric solar magnetic (GSM) coordinates if not specified otherwise. Auroral electrojet (AE) indices are obtained from the World Data Center in Kyoto, Japan. To eliminate small-scale structures, all data are averaged and resampled to a common time resolution of $1 \mathrm{~min}$. According to 
Baumjohann and Paschmann (1989) and Stiles et al. (1978) $P_{e} \ll P_{i}$ and $P_{e} \propto P_{i}$ in the plasma sheet, only ion data are used in this study (i.e., $P=P_{i}, n=n_{i}$ ).

\subsection{Selection criteria}

The identification of plasma sheet samples is based solely on magnetic field (and orbital) data. Following similar studies, e.g., Baumjohann and Paschmann (1989), the selection criteria are chosen to be the following:

1. Use data samples where both magnetic field and particle data are available.

2. a. $X_{\mathrm{GSM}} \leq-9 R_{\mathrm{E}}$,

b. $|Y|_{\mathrm{GSM}} \leq 15 R_{\mathrm{E}}$,

c. $|Z|_{\mathrm{GSM}} \leq 10 R_{\mathrm{E}}$.

3. a. $B_{x y} \leq 20 \mathrm{nT}$,

b. $\left|\angle\left(B_{y}, B_{x}\right)\right| \leq 15^{\circ}$,

c. $\angle\left(B_{z}, B_{x y}\right) \geq 15^{\circ}$.

The first criterion is necessary to make plasma sheet identification by FGM data and analysis reasonable. The orbital criteria define an approximate magnetotail plasma sheet box in which data are expected. Criterion (3a) ensures that the spacecraft is not too far away from the magnetic field reversal, (3b) limits the azimuthal distance (i.e., $Y_{\mathrm{GSM}}$ ) from the neutral sheet, and (3c) does so for the vertical (i.e., $Z_{\mathrm{GSM}}$ ) distance from the neutral sheet. To justify the selection of the above criteria, the plasma $\beta$ (the ratio of plasma and magnetic pressure) is calculated for each data point. A histogram of the resulting distribution is shown in Fig. 1. As can be seen, the vast majority of measurements feature a $\beta>0.5$, a typical identification criterion for plasma sheet samples ( $\beta>0.2$ was used in Zhu, 1990, indicated here by the vertical line). Therefore, the above criteria perform reasonably well. The dataset consists of 164262 individual measurements.

To further confirm the successful detection of plasma sheet samples, Fig. 2 displays the $Z_{\mathrm{GSM}}$ position of the individual measurements over time. Clearly, sub-annual variations can be detected in the time series. These variations stem from the definition of the GSM coordinate system and the precession of magnetic dipole axis during Earth's orbit around the Sun. Additionally, a long-term variation in the neutral sheet position can be observed. The period of this oscillation lies around 10-12 years. The resemblance with solar cycle periods will be the subject of further study.

The complete orbital distribution of measurements in the $X Z$ and $Y Z$ plane is shown in Fig. 3. For the nearEarth region, the data are quite evenly distributed around $Y_{\mathrm{GSM}}, Z_{\mathrm{GSM}}=0$. Further downtail, a strong bias towards geomagnetic south can be observed. Consequently, caution is needed when analyzing only data in these regions. For the total dataset, the measurements beyond $-12.5 R_{\mathrm{E}}$ make up only about $8 \%$ of all available samples.

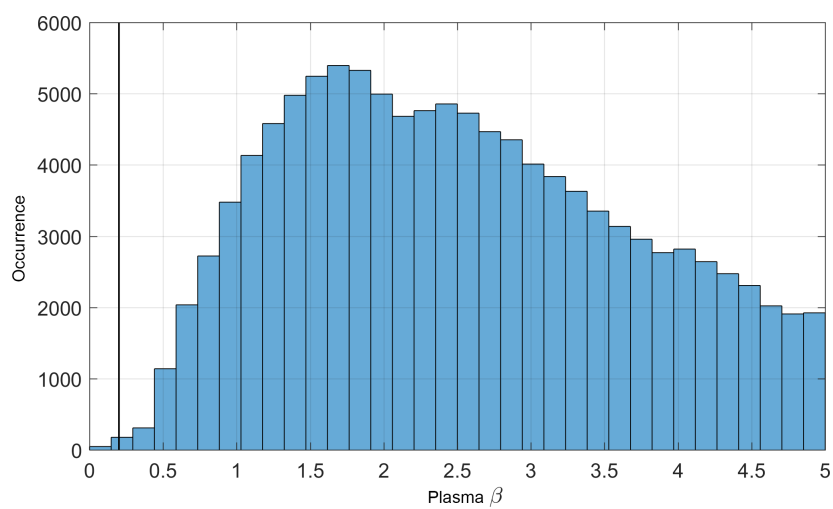

Figure 1. Occurrence of inferred plasma $\beta$ values in the complete dataset. The black vertical line indicates a value of $\beta=0.2$ as was used as the only plasma sheet criterion by Zhu (1990).

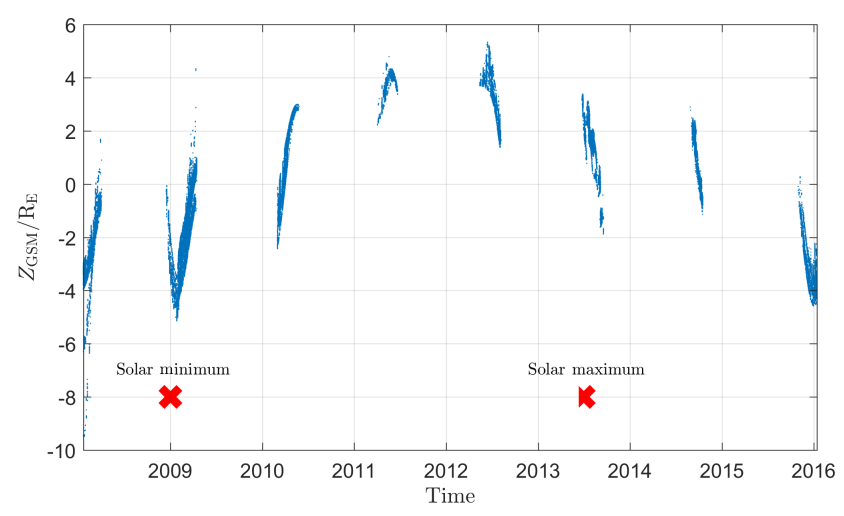

Figure 2. Variation in the inferred plasma sheet position over time. Each data point indicates a measurement made when THEMIS was located in the plasma sheet as identified by FGM data. The approximate positions of cycle 24 solar minimum and maximum are indicated as well.

\subsection{Data analysis procedure}

Following the observations of Zhu (1990), density and pressure relations will be used to determine an estimate for the polytropic index. According to Eq. (1), analyzing the data in $\log -\log$ space will yield a linear relationship, with the polytropic index as the slope between the two variables:

$\log P=\log \alpha+\gamma \log n$

Since a simple linear regression is based on the assumption that only the response variable is of nonzero error, it will not yield reasonable results. Both $P$ and $n$ feature measurement uncertainties. We will therefore adopt the method by Spaeth (1973), which is based on the minimization of the sum, $S$, of squared distances of the data points to the regression line 

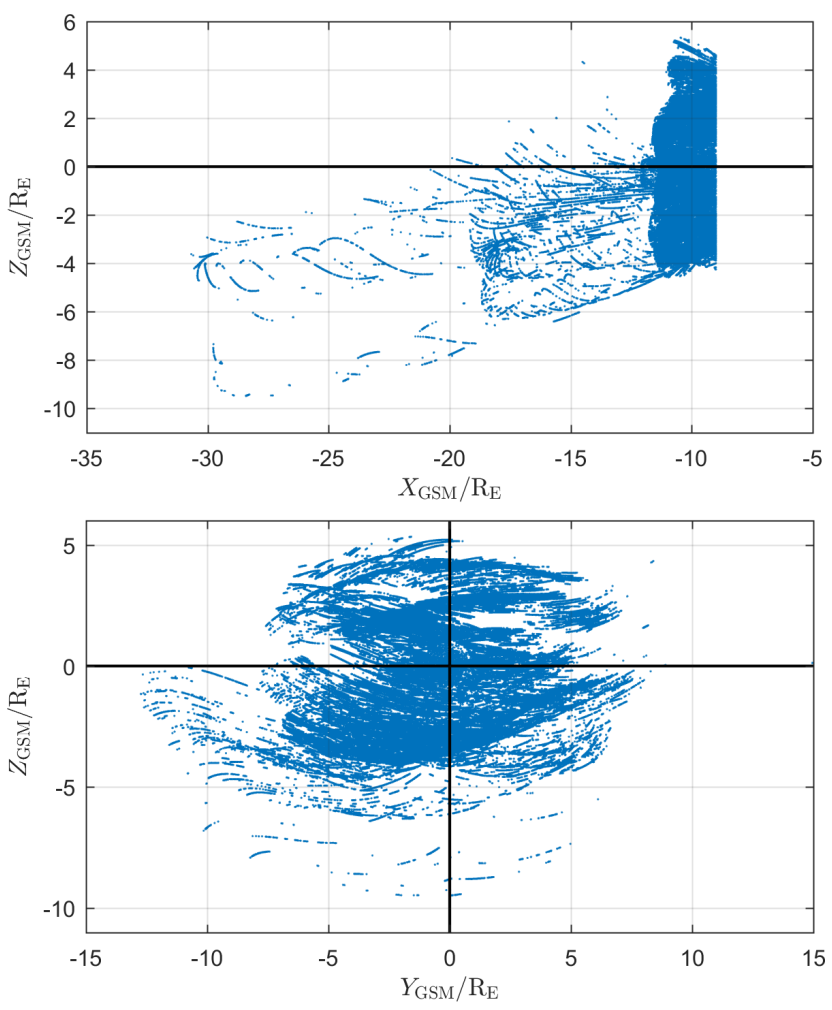

Figure 3. Orbital distribution of central plasma sheet data in the GSM frame. Blue dots indicate each individual 1 min measurement in the $X Z$ plane (upper panel) and $Y Z$ plane (lower panel). The solid black lines indicate GSM zero planes.

(see, e.g., Baumjohann and Paschmann, 1989):

$\min S(\gamma)=$

$\min \left(\frac{1}{\gamma^{2}+1} \sum_{k}\left[\log \left(P_{k} / \bar{P}\right)-\gamma \log \left(n_{k} / \bar{n}\right)\right]^{2}\right)$,

where the overbars denote geometric means of the relevant quantities. This procedure is also known as total leastsquares (TLS) method (see also Markovsky and Huffel, 2007).

\section{Results}

Following previous studies, the total database will be divided into subsets with respect to magnetospheric activity. In a second step, the analysis follows the ideas of Zhu (1990) - that is, a statistical analysis of individual spacecraft orbits will be performed.

The total dataset is displayed in Fig. 4. As can be observed the complete point cloud does not exhibit an obvious (only) linear relationship in the log-log space. This is especially quantified by the low correlation of $r^{2} \approx 0.31$. Still, even though the data are tremendously scattered, the huge number of samples results in a TLS fit of $\gamma \approx 1.722 \pm 0.015$

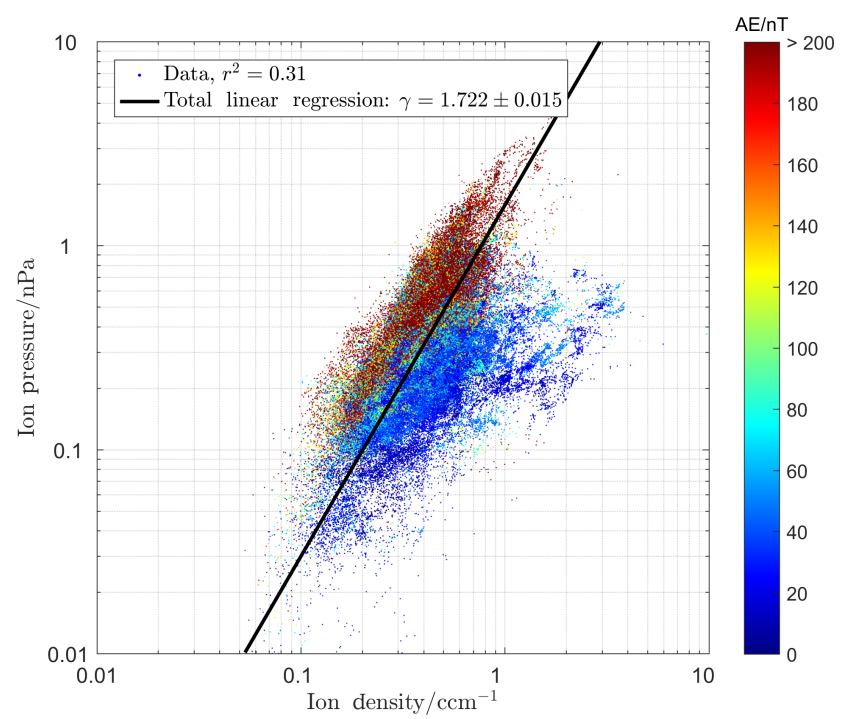

Figure 4. Scatter plot of all density and plasma pressure measurements. The total least-squares (TLS) fit comprises 164262 data points with $r^{2} \approx 0.31$. The data points are color-coded with respect to the corresponding AE index.

(95\% confidence), which is very close to an expected value of $\approx 5 / 3$.

Earlier studies have already emphasized the necessity to group the data according to magnetospheric activity, usually using the AE index as a proxy (Baumjohann and Paschmann, 1989; Goertz and Baumjohann, 1991; Pang et al., 2015). Therefore, in Fig. 4 a color code is incorporated indicating the related $\mathrm{AE}$ activity index for each measurement. The large amount of scattering stems from at least two different groups of samples that can be distinguished by the background magnetospheric activity.

Figure 5 shows the subset of data comprising $\mathrm{AE}>$ 200 nT. The samples now clearly exhibit a linear relationship (in $\log -\log$ ) with a correlation of $r^{2} \approx 0.70$. Describing the $n-P$ relation through the power law in Eq. (1) is therefore reasonable. The TLS fit gives $\gamma \approx 1.493 \pm 0.014$, which is very close to the results of Baumjohann and Paschmann (1989), Zhu (1990), Goertz and Baumjohann (1991), and Borovsky et al. (1998).

Using results of Frühauff and Glassmeier (2016), who compiled a database of THEMIS fast flow events in the magnetotail, we have scanned the data for measurements that have been made in the 1 min vicinity of an occurring fast flow (i.e., $V_{i} \geq 400 \mathrm{~km} \mathrm{~s}^{-1}$ ). Since the observation rate of fast flows is very low (see, e.g., Angelopoulos et al., 1992, Baumjohann et al., 1990, and Frühauff and Glassmeier, 2016), the number of detected fast flow encounters is of the order of $<10 \%$. Hence, they do not influence the analysis of the total dataset. However, analyzed separately, the observed $n-P$ observations show remarkable correlation $\left(r^{2} \approx 0.70\right)$, with a fitted polytropic index of $\gamma \approx 1.605 \pm 0.063$. 


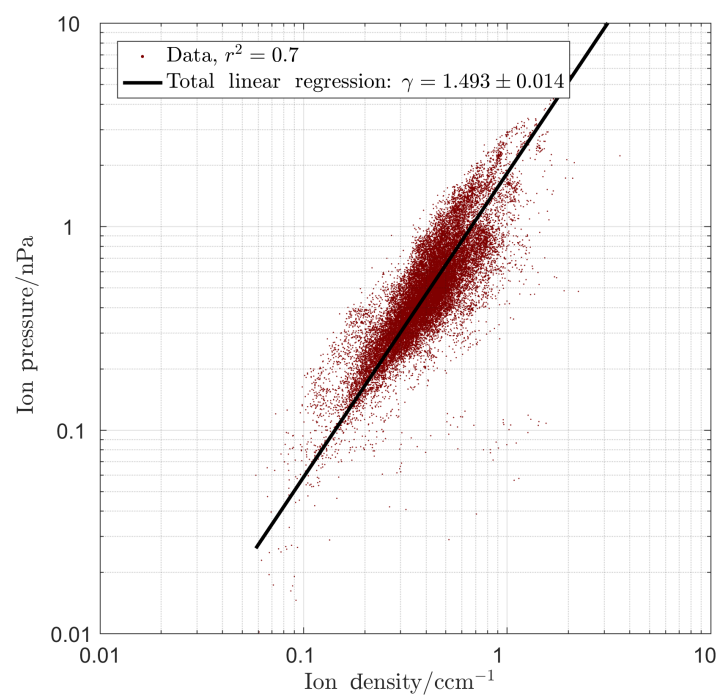

Figure 5. Scatter plot of density and plasma pressure measurements for data with $\mathrm{AE}>200 \mathrm{nT}$. The TLS fit comprises 26576 data points with $r^{2} \approx 0.7$.

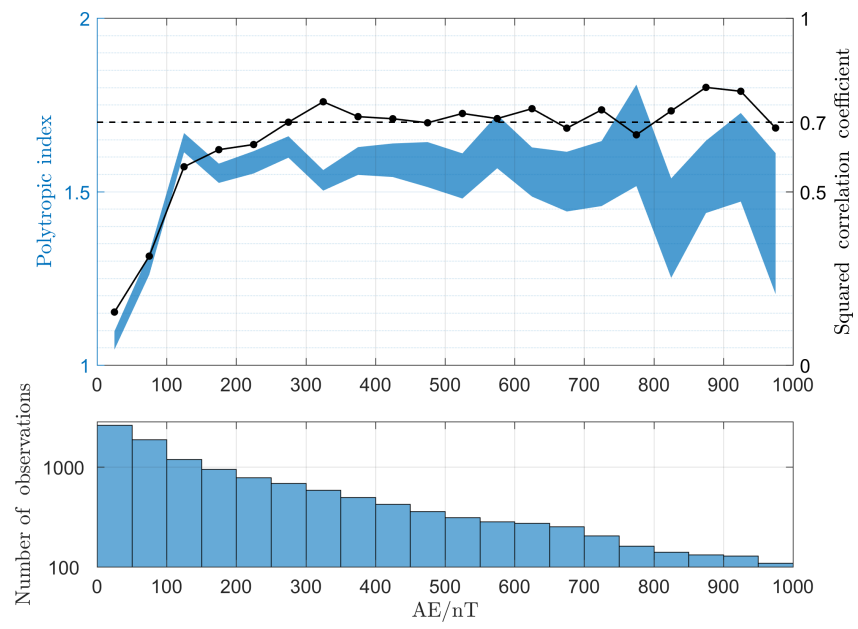

Figure 6. Fitted polytropic index as a function of AE index subsets. The upper panel shows the resulting polytropic index as its $95 \%$ confidence interval (blue) and the squared correlation coefficient (black). The lower panel displays the available number of observations for each subset.

Figure 4 indicates the existence of at least two, but potentially more subsets of data with respect to the $\mathrm{AE}$ index. Therefore, the database is divided into a range of $\mathrm{AE}$ intervals with a spacing of $50 \mathrm{nT}$. For the following analysis, only data with $X_{\mathrm{GSM}}>-12.5 R_{\mathrm{E}}$ to account for any possible bias introduced by the orbital coverage. For each subset the TLS procedure is applied. The results are shown in Fig. 6. As displayed by the bottom panel, each subset contains at least 100 individual samples. For all data with $\mathrm{AE}>250 \mathrm{nT}$ the correlation is around $r^{2} \approx 0.7$ or higher. For those intervals, the resulting mean polytropic index fit

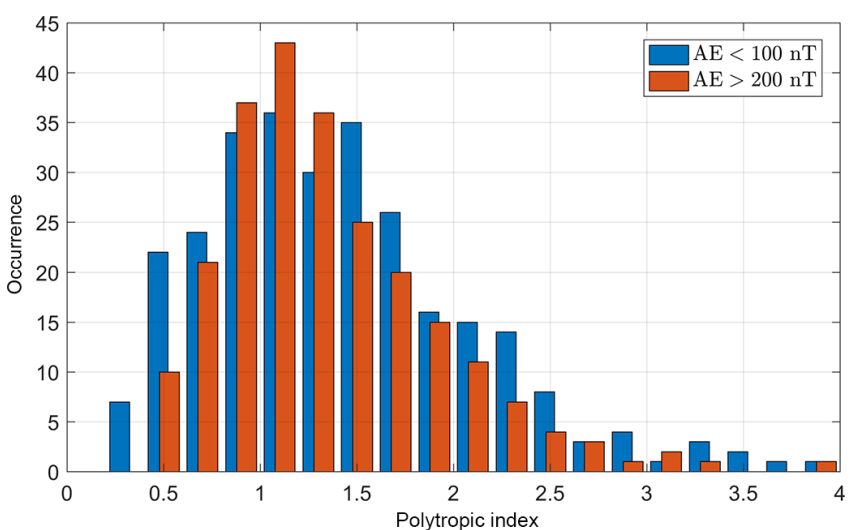

Figure 7. Distribution of polytropic indices for as fitted for each individual spacecraft orbit. The data are divided into low (blue) and high (orange) $\mathrm{AE}$ index subsets. For the chosen bin width $(\Delta \gamma=$ $0.2)$ the peaks of the distributions are located at $\gamma=1.1$ and $\gamma=1.5$ $(\mathrm{AE}<100 \mathrm{nT})$ and $\gamma=1.1(\mathrm{AE}>200 \mathrm{nT})$.

is around $\gamma \approx(1.56 \pm 0.08)$. No statistical significant deviations or trends can be observed. Below $\mathrm{AE}<200 \mathrm{nT}$ the correlation is very low, which can be attributed to data scattering as already observed in Fig. 4.

In contrast to the above procedure, several arguments indicate that the analysis needs to be performed on shorter timescales, i.e., to distinguish between individual spacecraft orbits, since the usual approach is to assume constant specific entropy for all observed flux tubes. Possible reasons for the violation of this assumption, and therefore data scattering, have been summarized by Zhu (1990), Liu and Rostoker (1990), and Goertz and Baumjohann (1991):

- The specific entropy of a flux tube crucially depends on its past.

- Flux tubes may thus contain different ratios of plasmas from different energetic regions, and may have been energized non-adiabatically by substorm activity or other mechanisms.

- Flux tubes may have lost particles through various processes during their evolution.

- For near-Earth plasma especially, initially hot flux tubes may have been loaded with cold ionospheric plasma, consequently changing their specific entropy.

- If any spacecraft were to only observe similar types of flux tubes during its orbits, scattering should be weak. Yet, the relative motion of a spacecraft across different flux tubes already causes scattering in the data.

Following the last argument, data scattering should be reduced by analyzing individual spacecraft orbits. For each of those time series, the spacecraft can still be expected to cross different flux tubes, but the deviations should be 
smaller. We have therefore first divided the initial database into the two obvious groups that can be seen in Fig. 4, namely $\mathrm{AE}<100 \mathrm{nT}$ and $\mathrm{AE}<200 \mathrm{nT}$. For each subset, TLS fitting is applied for the individual spacecraft orbits. The results are only considered if $r^{2} \geq 0.7$. The resulting histograms are shown in Fig. 7. The two distributions are very similar, both featuring mean values around $\gamma \approx 1.35$ with a standard deviation of $\sigma_{\gamma} \approx 0.68$. Consequently, no shift of the distribution towards lower polytropic indices as reported by previous works can be observed (Baumjohann and Paschmann, 1989; Goertz and Baumjohann, 1991). Instead, if analyzed individually, both active- and quiet-time distributions are similar and cover a wide range of polytropic indices (e.g., Pang et al., 2015). It should be noted that there is a small portion of events $(<1 \%)$ in the ranges $\gamma \rightarrow 0$ and $\gamma \rightarrow 4$. Since these events, by definition of the procedure, feature well-correlated data, this indicates that they might be of special physical nature. The reason for their fitted polytropic index being so different from the greater part of the distribution needs to be the subject of further study.

From Figs. 4 and 7 it can be inferred that, during both quiet and active times, the spacecraft observe flux tubes following a similar distribution of polytropic indices. Only during active times, though, do these flux tubes contain plasma of similar specific entropy as well (indicated by the smaller degree of deviation from a linear relationship in Fig. 4. The quiettime data feature a zoo of different (background) plasma portions with a large variety of specific entropies, leading to a high degree of scatter in the $n-P$ space.

\section{Radial and hemispheric dependence}

Although the radial range of THEMIS tail data is limited to either near-Earth space (i.e., $-10 R_{\mathrm{E}}$ ) or lunar orbit $\left(-60 R_{\mathrm{E}}\right)$, we follow the suggestions of Borovsky et al. (1998) to analyze the radial distribution of polytropic indices.

Figure 9 shows the fitted polytropic index for a range of bins in $X_{\mathrm{GSM}}$ direction along the magnetotail. For the correlation of the subsets of data to be meaningful, we include only the magnetospheric active data according to $\mathrm{AE}>$ $200 \mathrm{nT}$. Still, as can be observed in the plot, the correlation for data beyond $-20 R_{\mathrm{E}}$ is very weak, making the fitting process insignificant. Even for the near-Earth data, the squared correlation coefficient rarely reaches 0.7 , making the interpretation of the parameters difficult. For the bins in the regions -20 to $-16 R_{\mathrm{E}}$ and $-11 R_{\mathrm{E}}$ a polytropic index close to adiabaticity is derived. However, around $-13 R_{\mathrm{E}}$ a clear depression can be observed. The fit results in a polytropic index close to the isothermal regime, indicating energy loss of the plasma in this region. This result indicates a possible connection to the flow-braking region which is usually found at similar downtail distances (see, e.g. Baumjohann and Glassmeier, 1984, Shiokawa et al., 1997, Dubyagin et al., 2010, and Yao et al., 2012 for observations and possible mechanisms).

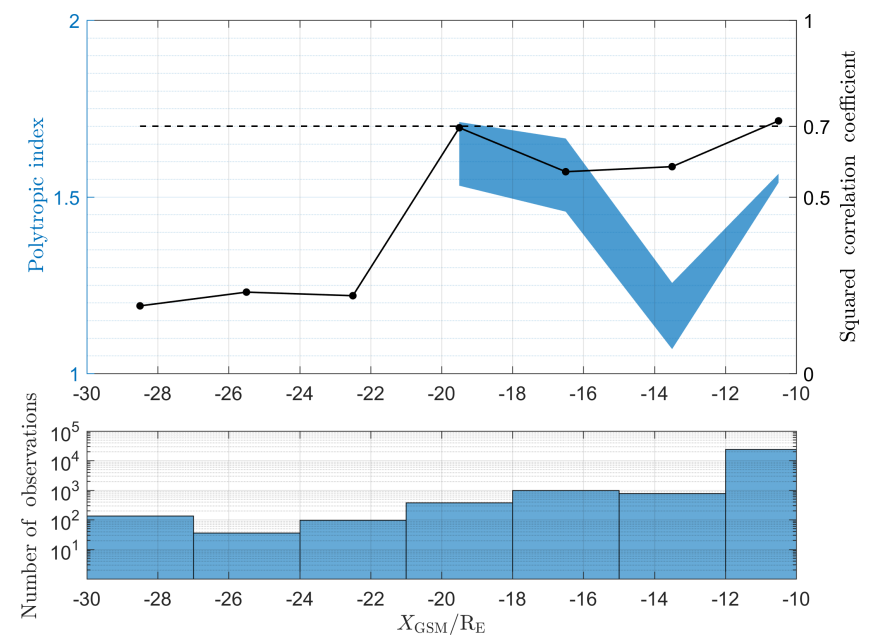

Figure 8. Radial dependence of the complete dataset for which $\mathrm{AE}>200 \mathrm{nT}$. The upper panel shows the $95 \%$ confidence interval of the fitted polytropic index (blue) and the squared correlation coefficient (black). The lower panel displays the available number of observation for each bin.

To study possible hemispheric differences in the $Y$ and $Z$ directions of the tail, the orbit-wise analysis is carried out in the relevant hemispheres. Each orbit for which the squared correlation exceeds 0.7 is assigned to the corresponding region if all of its data points fulfills one of the following criteria:

$$
\begin{aligned}
B_{X, \mathrm{GSM}} & >5 \mathrm{nT} \rightarrow \text { northern magnetospheric hemisphere; } \\
B_{X, \mathrm{GSM}} & <5 \mathrm{nT} \rightarrow \text { southern magnetospheric hemisphere; } \\
Y_{\mathrm{GSM}} & >1 R_{\mathrm{E}} \rightarrow \text { duskside hemisphere; } \\
Y_{\mathrm{GSM}}<-1 R_{\mathrm{E}} & \rightarrow \text { dawnside hemisphere. }
\end{aligned}
$$

Please note that magnetic field data are used to distinguish between northern and southern hemispheres, since the GSM coordinates may not always be a sufficient measure of neutral sheet distance. In case of dawn-dusk hemispheres, no similar condition can be derived from magnetic field data. Furthermore, $Y_{\mathrm{GSM}}$ is expected to work reasonably well. The resulting distributions are shown in Figs. 9 and 10. Similar to Fig. 7 the spread of the fitted polytropic indices is large. At the same time, the mean values of the different regions are not significantly different. We conclude that no obvious north-south or dawn-dusk symmetries can be observed in the data.

\section{Comparison with ARTEMIS data}

As a matter of completeness, the data from the ARTEMIS part of the mission are analyzed as well. The data selection criteria are equal to those described in Sect. 2.1 with the addition of the following orbit requirement:

4. IF $X_{\mathrm{SSE}}<0$ THEN $Y Z_{\mathrm{SSE}} \geq 1 R_{\mathrm{M}}$, 




Figure 9. Dawn-dusk hemispheric dependence of near-Earth data (i.e., $X_{\mathrm{GSM}}>-12.5 R_{\mathrm{E}}$ ). The hemispheres are defined through $|Y|_{\mathrm{GSM}}>1 R_{\mathrm{E}}$.

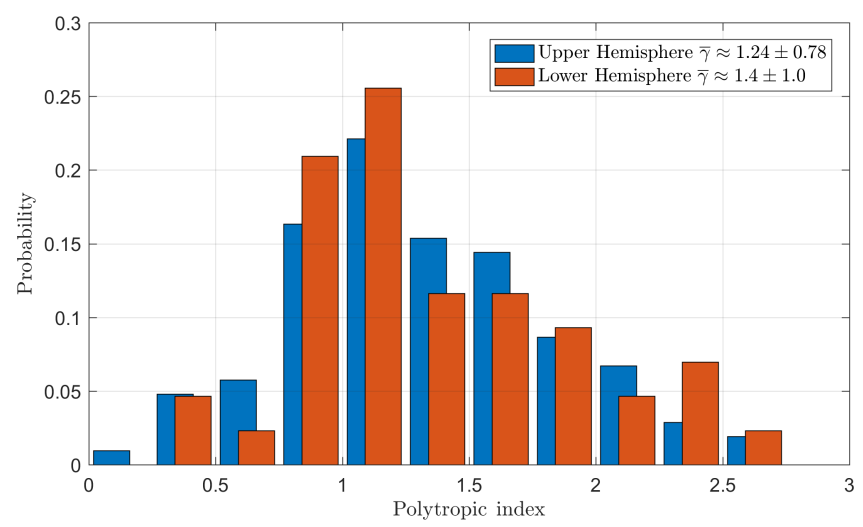

Figure 10. North-south hemispheric dependence of near-Earth data (i.e. $X_{\mathrm{GSM}}>-12.5 R_{\mathrm{E}}$ ). The hemispheres are defined through $\left|B_{x}\right|_{\mathrm{GSM}}>5 \mathrm{nT}$.

where the orbital information is based on selenocentric solar ecliptic (SSE) coordinates, similar to the geocentric solar ecliptic (GSE) definition with the exception of having the Moon's center at the origin, and the Moon's radius, $R_{\mathrm{M}}$. This additional requirement ensures that eclipse data, i.e., spacecraft shadow passes, are not included in the dataset. The resulting orbital distribution covers ranges of $X_{\mathrm{GSM}}$ between -66 and $-52 R_{\mathrm{E}}$.

The total linear regression of the data is shown in Fig. 11. Similar to the THEMIS data, a slight grouping of subsets can be observed considering the $\mathrm{AE}$ index. However, a very clear linear relationship of data during strong magnetospheric activity is not evident. Again, fitting the whole dataset results in a very poor correlation of $r^{2} \approx 0.34$ with an estimated polytropic index of $\gamma \approx 1.566 \pm 0.029$.

The analysis of individual spacecraft orbits is displayed in Fig. 12. The histogram is qualitatively very similar to Fig. 7, again featuring a maximum between $\gamma=1$ and 2. However, since the number of detected orbits is much lower in case of ARTEMIS, the statistics are very poor. From this analysis no

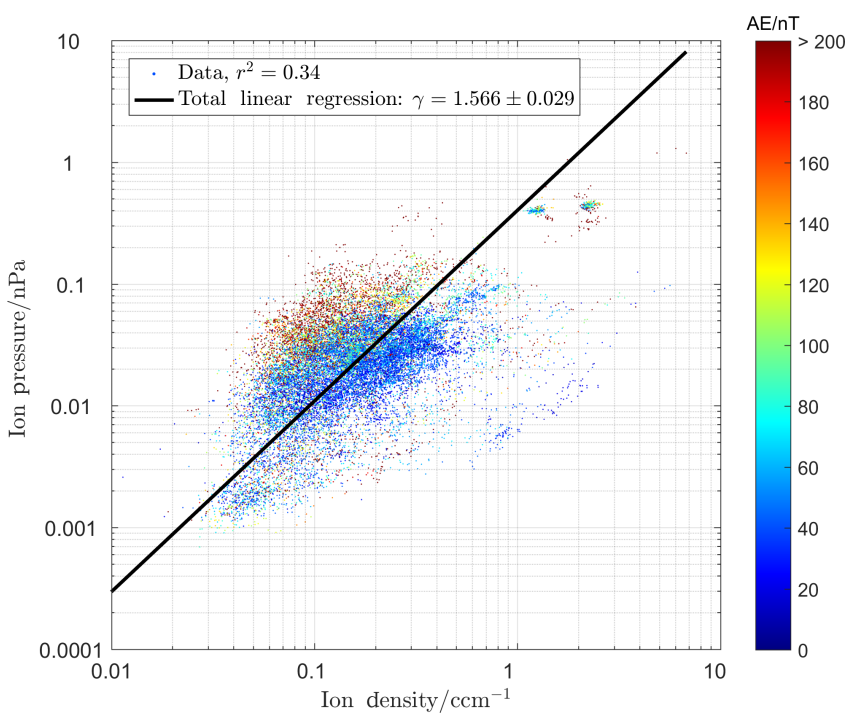

Figure 11. Scatter plot of all density and plasma pressure measurements with the ARTEMIS probes. The TLS fit comprises 21054 data points with $r^{2} \approx 0.34$. The data points are color-coded with respect to the corresponding $\mathrm{AE}$ index.

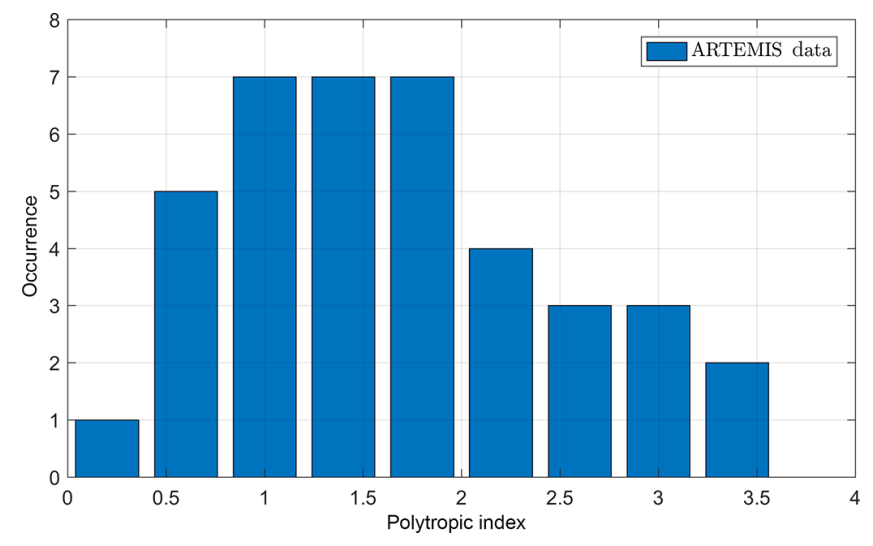

Figure 12. Distribution of polytropic indices for as fitted for each individual spacecraft orbit. The fit is considered successful if $r^{2} \geq$ 0.7 .

obvious difference between near-Earth and downtail regions can be inferred.

\section{Summary}

This study revisits the observational determination of the polytropic index in the Earth's magnetotail using data from the THEMIS and ARTEMIS missions. Taking the usual approach (Baumjohann and Paschmann, 1989; Zhu, 1990; Liu and Rostoker, 1990; Goertz and Baumjohann, 1991), i.e., fitting a power law equation of state to the total point cloud of density and pressure observations, the results indicate a polytropic index very close to an expected adiabatic index 
of $\gamma=5 / 3$. Yet, the amount of scattering and the poor linear correlation of the data indicate that this simple approach does not yield useful results.

The addition of AE index data as a proxy for magnetospheric activity shows that the near-Earth point cloud in the $n-P$ space is actually made from two separate distributions, one for the quiet-time magnetotail and one for an active magnetotail with $\mathrm{AE}>200 \mathrm{nT}$. While the former point cloud still displays a large amount of data scattering with a polytropic index clearly below adiabatic behavior, the active magnetotail data are well correlated with a fitted polytropic index of $\gamma \approx 1.5$. A similar result is obtained when analyzing the polytropic indices for smaller subsets of data with respect to their activity index (see Fig. 6). These results compare well to previous studies such as Baumjohann and Paschmann (1989), Zhu (1990), Goertz and Baumjohann (1991). The analysis of data during fast flow events only results in a small dataset with highly correlated density and pressure variations. The fitted polytropic index is very close to the adiabatic value.

While it is tempting to conclude that the active magnetotail is quasi-adiabatic and the quiet-time tail is not, one has to be careful about the actual cause of data scattering in the point cloud. Since the analysis incorporates data of a variety of locations within the plasma sheet and might contain a zoo of dynamic structures intrinsic to the tail, it seems natural to assume that the contributions to the point cloud feature different specific entropies, leading to a vertical offset in the $n-P$ space, while at the same time indeed showing adiabatic behavior when considered separately. To summarize the arguments in Sect. 3, the spacecraft will have crossed a variety of different flux tubes, each with different specific entropy, which mainly depends on the flux tube's past. Each of these may have undergone heating, energy loss, or even particle loss during its passage through the tail. Therefore, the assumption of the total linear regression, namely that the observed specific entropy of the flux tubes is approximately constant, can not be justified.

Consequently, and as already suggested by others, such as Zhu (1990), Liu and Rostoker (1990), and Goertz and Baumjohann (1991), an analysis on smaller timescales can be performed by fitting individual polytropic indices for each spacecraft orbit (see Fig. 7). For a large number of orbits, the data correlation is high enough to be incorporated into a histogram. The distribution of polytropic indices is very similar for both quiet and active magnetotail. This result is in contrast to the interpretation of the total least-squares fit only.

The main findings of this work can be summarized as follows:

1. Both quiet and active time plasma sheets show a population of flux tubes with similar polytropic indices. This means that, here, the quiet tail is not different from the active tail. However, since data scattering in the quiet- time tail is higher, the distributions of specific entropies must be different from one another.

2. Data during fast flows show remarkable correlation with a polytropic index very close to $5 / 3$.

3. The total distribution of polytropic indices is broad, with approximately $68 \%$ of all indices between $\gamma=$ 0.67 and $\gamma=2$. Clearly, the magnetotail features processes that show all different kinds of thermodynamic scenarios leading to an average polytropic index between isothermal and adiabatic.

4. Some of the events found in the THEMIS data seem to be of very different physical nature. Their properties need to be evaluated in detail in future studies.

5. The spatial distribution of polytropic indices through the magnetotail reveals a depression region around $-13 R_{\mathrm{E}}$ where close-to-isothermal behavior is observed in the plasma data. This observation is possibly related to flow-braking mechanisms in the dipole region of the magnetotail.

6. No asymmetries between north-south or dawn-dusk regions can be reported.

7. The addition of data from the ARTEMIS mission and, therefore, downtail region of the plasma sheet indicates a very similar behavior. However, due to the limited number of available orbits that satisfy the criteria, a quantitative analysis based on THEMIS data alone remains difficult.

A thermodynamic closure equation like Eq. (1) together with the assumption of adiabatic behavior is input to many magnetospheric and magnetotail models and magnetohydrodynamic simulations. The analysis of THEMIS data on individual orbits indicates that many different values of the polytropic index are determined when traversing different flux tubes in the plasma sheet. Therefore, the validity of a single polytropic index as input to models and simulations seems questionable. Since heat flux or exchange appears to be common even in quiet times, application of this simplification might change the outcome to certain extent. Whether or not the relative change of the results is substantial is certainly a question best to be answered by theoretical considerations. A first step into this direction has been done by Livadiotis (2016), who aims at a description of a superposition of polytropic indices in the heliosheath. Our results indicate that a similar treatment should be considered when modeling magnetospheric regions, such as the magnetotail. 


\section{Data availability}

THEMIS data and the latest calibration files are publicly available at http://themis.ssl.berkeley.edu/ or via the SPEDAS software. Auroral electrojet data can be found at http://wdc.kugi.kyoto-u.ac.jp/.

Competing interests. The authors declare that they have no conflict of interest.

Acknowledgements. Auroral electrojet indices for all necessary events were obtained from the World Data Center in Kyoto, Japan. We acknowledge NASA contract NAS5-02099 and V. Angelopoulos for use of data from the THEMIS Mission. Specifically: C. W. Carlson and J. P. McFadden for use of ESA data and D. Larson for use of SST data. This project is financially supported by the German Ministerium für Wirtschaft und Energie and the Deutsches Zentrum für Luft- und Raumfahrt under contract 50 OC 1403.

The topical editor, C. Owen, thanks the two anonymous referees for help in evaluating this paper.

\section{References}

Angelopoulos, V.: The THEMIS Mission, Space Sci. Rev., 141, 534, 2008.

Angelopoulos, V.: The ARTEMIS Mission, Space Sci. Rev., 165, 3-25, doi:10.1007/s11214-010-9687-2, 2011.

Angelopoulos, V., Baumjohann, W., Kennel, C. F., Coronti, F. V., Kivelson, M. G., Pellat, R., Walker, R. J., Luehr, H., and Paschmann, G.: Bursty bulk flows in the inner central plasma sheet, J. Geophys. Res.-Space, 97, 4027-4039, doi:10.1029/91JA02701, 1992.

Auster, H. U., Glassmeier, K. H., Magnes, W., Aydogar, O., Baumjohann, W., Constantinescu, D., Fischer, D., Fornacon, K. H., Georgescu, E., Harvey, P., Hillenmaier, O., Kroth, R., Ludlam, M., Narita, Y., Nakamura, R., Okrafka, K., Plaschke, F., Richter, I., Schwarzl, H., Stoll, B., Valavanoglou, A., and Wiedemann, M.: The THEMIS Fluxgate Magnetometer, Space Sci. Rev., 141, 235-264, doi:10.1007/s11214-008-9365-9, 2008.

Baumjohann, W. and Glassmeier, K.-H.: The transient response mechanism and Pi2 pulsations at substorm onset - Review and outlook, Planet. Space Sci., 32, 1361-1370, doi:10.1016/00320633(84)90079-5, 1984

Baumjohann, W. and Paschmann, G.: Determination of the polytropic index in the plasma sheet, Geophys. Res. Lett., 16, 295298, doi:10.1029/GL016i004p00295, 1989.

Baumjohann, W., Paschmann, G., Sckopke, N., Cattell, C. A., and Carlson, C. W.: Average ion moments in the plasma sheet boundary layer, J. Geophys. Res.-Space, 93, 11507-11520, doi:10.1029/JA093iA10p11507, 1988.

Baumjohann, W., Paschmann, G., and Luehr, H.: Characteristics of high-speed ion flows in the plasma sheet, J. Geophys. Res.Space, 95, 3801-3809, doi:10.1029/JA095iA04p03801, 1990.

Borovsky, J. E., Thomsen, M. F., Elphic, R. C., Cayton, T. E., and McComas, D. J.: The transport of plasma sheet material from the distant tail to geosynchronous orbit, J. Geophys. Res.-Space, 103, 20297-20332, doi:10.1029/97JA03144, 1998.

Dubyagin, S., Sergeev, V., Apatenkov, S., Angelopoulos, V., Nakamura, R., McFadden, J., Larson, D., and Bonnell, J.: Pressure and entropy changes in the flow-braking region during magnetic field dipolarization, J. Geophys. Res.-Space, 115, A10225, doi:10.1029/2010JA015625, 2010.

Frühauff, D. and Glassmeier, K.-H.: Statistical analysis of magnetotail fast flows and related magnetic disturbances, Ann. Geophys., 34, 399-409, doi:10.5194/angeo-34-399-2016, 2016.

Goertz, C. K. and Baumjohann, W.: On the thermodynamics of the plasma sheet, J. Geophys. Res.-Space, 96, 20991-20998, doi:10.1029/91JA02128, 1991.

Huang, C. Y., Goertz, C. K., Frank, L. A., and Rostoker, G.: Observational determination of the adiabatic index in the quiet time plasma sheet, Geophys. Res. Lett., 16, 563-566, doi:10.1029/GL016i006p00563, 1989.

Kivelson, M. G. and Spence, H. E.: On the possibility of quasistatic convection in the quiet magnetotail, Geophys. Res. Lett., 15, 1541-1544, doi:10.1029/GL015i013p01541, 1988.

Liu, W. W. and Rostoker, G.: Ionospheric outflow of plasma and compression relationship in the plasma sheet, Geophys. Res. Lett., 17, 1849-1852, doi:10.1029/GL017i011p01849, 1990.

Livadiotis, G.: Superposition of Polytropes in the Inner Heliosheath, Astrophys. J. Suppl. S., 223, 13, doi:10.3847/00670049/223/1/13, 2016.

Markovsky, I. and Huffel, S. V.: Overview of total leastsquares methods, Signal Process., 87, 2283-2302, doi:10.1016/j.sigpro.2007.04.004, 2007.

McFadden, J., Carlson, C., Larson, D., Bonnell, J., Mozer, F., Angelopoulos, V., Glassmeier, K.-H., and Auster, U.: THEMIS ESA First Science Results and Performance Issues, Space Sci. Rev., 141, 477-508, 2008a.

McFadden, J. P., Carlson, C. W., Larson, D., Ludlam, M., Abiad, R., Elliott, B., Turin, P., Marckwordt, M., and Angelopoulos, V.: The THEMIS ESA Plasma Instrument and In-flight Calibration, Space Sci. Rev., 141, 277-302, doi:10.1007/s11214-008-9440-2, 2008 b.

Nishida, A.: The Earth's Dynamic Magnetotail, Space Sci. Rev., 91, 507-577, doi:10.1023/A:1005223124330, 2000.

Pang, X., Cao, J., Liu, W., Ma, Y., Lu, H., Yang, J., Li, L., Liu, X., Wang, J., Wang, T., and Yu, J.: Polytropic index of central plasma sheet ions based on MHD Bernoulli integral, J. Geophys. Res.-Space, 120, 4736-4747, doi:10.1002/2014JA020855, 2015.

Shiokawa, K., Baumjohann, W., and Haerendel, G.: Braking of high-speed flows in the near-Earth tail, Geophys. Res. Lett., 24, 1179-1182, doi:10.1029/97GL01062, 1997.

Siscoe, G. L.: Solar system magnetohydrodynamics, in: SolarTerrestrial Physics: Principles and Theoretical Foundations, edited by: Carovillano, R. L. and Forbes, J. M., vol. 104 of Astrophysics and Space Science Library, 11-100, doi:10.1007/97894-009-7194-3_2, 1983.

Spaeth, H.: Algorithmen für elementare Ausgleichsmodelle, Verfahren der Datenverarbeitung, Oldenbourg, Munich, 1973.

Spence, H. E. and Kivelson, M. G.: The variation of the plasma sheet polytropic index along the midnight meridian in a finite width magnetotail, Geophys. Res. Lett., 17, 591-594, doi:10.1029/GL017i005p00591, 1990. 
Stiles, G. S., Hones Jr., E. W., Bame, S. J., and Asbridge, J. R.: Plasma sheet pressure anisotropies, J. Geophys. Res.-Space, 83, 3166-3172, doi:10.1029/JA083iA07p03166, 1978.

Yao, Z. H., Pu, Z. Y., Fu, S. Y., Angelopoulos, V., Kubyshkina, M., Xing, X., Lyons, L., Nishimura, Y., Xie, L., Wang, X. G., Xiao, C. J., Cao, X., Liu, J., Zhang, H., Nowada, M., Zong, Q. G., Guo, R. L., Zhong, J., and Li, J. X.: Mechanism of substorm current wedge formation: THEMIS observations, Geophys. Res. Lett., 39, L13102, doi:10.1029/2012GL052055, 2012.
Zhu, X. M.: Plasma sheet polytropic index as inferred from the FPE measurements, Geophys. Res. Lett., 17, 2321-2324, doi:10.1029/GL017i013p02321, 1990. 